\section{Regula Spreyermann}

\section{Häufige Schmerzsyndrome bei Menschen mit Behinderung}

- Schmerzen am Bewegungsapparat, wie sie durch chronische Überbelastung zustande kommen können - beispielsweise die Schulterschmerzen bei Rollstuhlfahrern oder die Schulter-, Nacken- und Ellbogenschmerzen beim Gehen an Stöcken.

- Rückenschmerzen, die durch Fehlhaltungen auftreten - sei es im Rahmen einer Wirbelsäulenverletzung oder bei Skoliose oder auch sekundär, z.B. durch eine falsche Sitzposition im Rollstuhl.

- Neuropathische Schmerzen, die durch Rückenmarksschädigung auftreten - sei es auf Höhe der Lähmung, unterhalb des Lähmungsniveaus oder im Rahmen einer Schädigung des Gehirns.

- Schmerzen durch die Tonuserhöhung der Muskulatur bzw. einschiessende Spastik.

- Periphere Kompressionssyndrome wie z.B. das Karpaltunnelsyndrom, das ebenfalls durch das Rollstuhlfahren, das Transferieren, aber auch das Gehen an Stöcken gehäuft auftritt.

- Schmerzen im Rahmen der stark gehäuft auftretenden Osteoporose und zusätzlich bei den dadurch bedingten Frakturen sowie Schmerzen durch weitere Komplikationen wie die Reflexdystrophie usw.

- Schmerzen durch Entwicklung einer somatoformen Schmerzstörung.

\title{
Schmerzen bei Behinderung: Mehr als doppelte Last!
}

\section{Ganzheitliche Behandlungsansätze in der Schmerztherapie}

Liegt die Häufigkeit von Menschen mit schweren und mittelschweren Schmerzen in der Schweiz und unseren Nachbarländern mit 15-17\% schon hoch (jede/r Sechste ist betroffen), so erschreckt umso mehr, wie häufig Menschen mit Behinderung an Schmerzen leiden: 70-80\% der Querschnittgelähmten [1, 2] und wahrscheinlich ähnlich viele Menschen mit Cerebralparese oder ähnlicher Symptomatik sind betroffen.

- Alle anderen Schmerzen, die auch Nicht-Behinderte im Laufe des Lebens entwickeln können.

Kurzum: Die Häufigkeit von 70-80\% Schmerzen bei Menschen mit Querschnittlähmung bzw. ähnlicher Behinderung [3] drücken aus, dass es um eine Summation geht (Abb. 1). Und umgekehrt: Diese zusätzlichen Schmerzen stellen natürlich auch eine zusätzliche Behinderung dar - sie beeinträchtigen Aktivität sowie Partizipation im Alltag und führen oft zu verstärkter Abhängigkeit, Depression und sozialem Rückzug. Auch hier handelt es sich um eine Summation.

\section{Neuropathische Schmerzen -} besonders «nervig»

Wer selbst einmal an neuropathischen Schmerzen gelitten hat, erinnert sich gewiss, wie unangenehm, quälend und «unüberhörbar» solche Schmerzen sind. Sie «nerven»! Und sie nerven auch in der Behandlung: Trotz einer breiten Palette von Medikamenten hilft oft einfach keines, während die Schmerzen unerträglich bleiben. Dies zeigt sich auch in den folgenden Zahlen: Ungenügend behandelte neuropathische Schmerzen machen 20\% einer Schmerzambulanz aus - und Menschen mit chronischen neuropathischen Schmerzen suchen oft jahrelang nach einer geeigneten Therapie und wechseln über 10 Jahre hinweg durchschnittlich 8-mal (!) den Arzt [4].

Behandlung: Unbedingt, aber nicht zu jedem Preis!

Selbstverständlich verfügen wir angesichts der Häufigkeit der Schmerzsyndrome in unserer Klinik über die

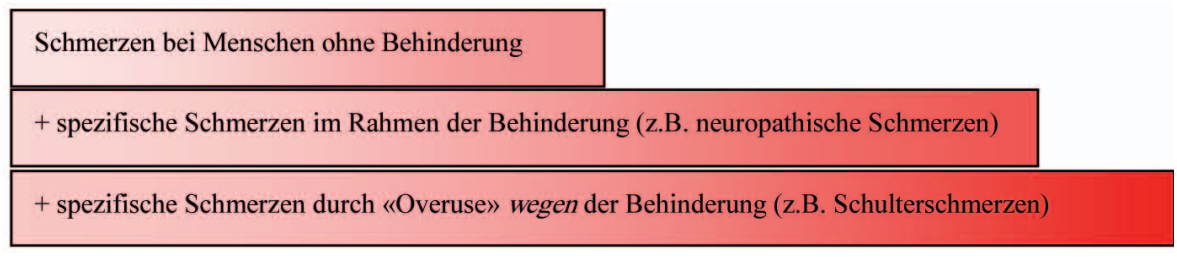

Abb. 1. Summation der Schmerzen.

\section{KARGER}

Fax +497614520714 Information@Karger.d Information@Kar
๑) 2012 S. Karger GmbH, Freiburg

Accessible online at: www.karger.com/sz
Dr. med. Regula Spreyermann

REHAB Basel, Zentrum für Querschnittgelähmte und Hirnverletzte

Schweizerisches Paraplegikerzentrum Basel

Im Burgfelderhof 40, 4012 Basel, Schweiz

Tel. +41 61-3250010, Fax -3250121

r.spreyermann@rehab.ch 
ganze Palette der herkömmlichen Schmerztherapien, einschliesslich konsiliarisch der Behandlung durch unsere Neurologen und extern interventionellen Schmerzspezialisten. Allerdings zeigen sich bei der Langzeitbetreuung von Menschen mit Querschnittlähmung ein paar Besonderheiten, die ich hier kurz nennen möchte, weil sie unsere Behandlungsstrategie wesentlich beeinflusst haben.

Menschen mit Querschnittlähmung müssen zur Behandlung der Spastik und der Blasen-/Darmfunktionsstörung bereits einige Medikamente täglich regelmässig einnehmen; oft sind sie noch im erwerbstätigen Alter, fühlen sich gesund und möchten bei Schmerzen nicht ein Medikament einnehmen müssen, das sie zusätzlich beeinträchtigt. Genau das ist aber aufgrund der Nebenwirkungen der zentral wirkenden Antiepileptika, die wir zur Behandlung neuropathischer Schmerzen einsetzen, der Fall. Dazu kommt, dass die durch die Rückenmarkstraumatisierung entstandenen, neuropathischen Schmerzen meist andauernd vorhanden sind, d.h., dass es um eine Dauerbehandlung geht, und da befürchten unsere Patienten oft eine Leberschädigung oder andere negative Auswirkungen.

Und wie oben bereits erwähnt: Oft helfen unsere herkömmlichen Medikamente selbst beim Einsatz hoher Dosen oder intrathekaler Gabe einfach nicht oder nur ungenügend. Teilweise besteht dadurch eine verstärkte Spastik, die zusätzlich belastet - oder umgekehrt ist die Spastik schmerzhaft. Zudem haben wir leider auch oft Patienten mit komplexen, seit Jahrzehnten bestehenden, chronifizierten Schmerzen, die aus mehreren Schmerzarten und -regionen bestehen.

Aus all diesen Gründen haben wir schon vor über 10 Jahren ein komplementärmedizinisches Behandlungsangebot in unserem Ambulatorium aufgebaut, das wir gezielt zur Schmerzbehandlung miteinsetzen.

\section{Komplementärmedizin} bei chronischen Schmerzen

Was bringt die Komplementärmedizin bei chronischen Schmerzen? Das wollten wir natürlich auch wissen! Wir haben von Anfang an gemeinsam mit unseren Patienten individualisierte Schmerzprotokolle angefertigt und sie genau und wiederholt darin instruiert, das Ansprechen auf eine unserer sequenziell eingesetzten komplementärmedizinischen Therapien zu dokumentieren. Durch regelmässige Evaluation wechselten wir die Behandlungsmethode, wenn sich nach 6 Wochen keine Besserung abzeichnete. Damals boten wir Homöopathie, Neuraltherapie und Akupunkturmassage nach Penzel an. Das Ziel war, einerseits die Wirksamkeit der Behandlungen darzulegen, andererseits aber auch Hinweise bezüglich der Indikationsstellung zu erhalten: Welche Methode ist bei welchen Problemen am besten einzusetzen? Tatsächlich fanden wir - wenn auch bei beschränkten Patientenzahlen -, dass Neuraltherapie bei Schmerzen am Bewegungsapparat in über $90 \%$ eine Besserung bringt, während die Homöopathie zwar oft den Schmerz nicht wesentlich verringerte, dafür aber die häufige Begleitsymptomatik [5] wie depressive Verstimmung, Schlafstörungen oder vegetative Symptome deutlich verbesserte. Der Vollständigkeit halber sei erwähnt, dass - trotz aller Überzeugungsarbeit nur die Hälfte der Schmerzprotokolle auswertbar war, meist durch unüberwindbare Probleme beim Rating bedingt.

\section{Ansprechen auf verschiedene Behandlungsmethoden}

Interessanterweise zeigte sich bei dieser Auswertung im Jahr 2004 auch, dass einige unserer Patienten sehr wohl auf eine der drei Methoden ansprachen, auf eine andere aber gar nicht. Und es zeigte sich auch, dass durch unsere sequenziell durchgeführten Therapien wohl wichtige Hinweise zum Ansprechen bei verschiedenen Symptomen gewonnen werden konnten, die Patienten andererseits aber oft auch die Geduld verloren und die Behandlung abbrachen.

2005 haben wir deshalb unser Konzept geändert und sind zur komplementärmedizinischen Komplexbehandlung übergegangen: Anhand einer eingehenden Erstbesprechung durch den komplementärmedizinisch versierten Arzt wurde festgelegt, welche Problembereiche vorliegen und wie diese angegangen werden sollen: parallel oder nacheinander - und womit. Fast immer wurden Phytotherapie, eine physiotherapeutische osteopathische Behandlung und Neuraltherapie oder Akupunktur eingesetzt, bei Bedarf begleitet von Homöopathie. Bei einer Besserung wurden die Behandlungsintervalle verlängert.

\section{Das Konzept unserer heutigen Schmerzsprechstunde}

Durch die intensive Begleitung unserer Patienten im Rahmen der Nachsorge und der Schmerzbehandlung (Abb. 2) hat sich in den letzten Jahren unser Behandlungskonzept laufend verändert: Es setzt stark auf die partnerschaftliche Zielsetzung gemeinsam mit den Patienten und baut für die Zielerreichung auf ein interprofessionelles Behandlungsteam. Noch zuvor aber ist der Aufbau einer tragfähigen Beziehung zwischen den Ärzten der Schmerzsprechstunde und den Patienten zentral, um - nach oft vielen enttäuschend verlaufenen Behandlungsversuchen - das notwendige Vertrauen und die Motivation für den neuen Behandlungsversuch aufzubauen. Dies bedeutet, viel Zeit für das sorgfältige Aktenstudium, für Anamnese und Untersuchung [5, 6], für Aufklärung und sorgfältige 


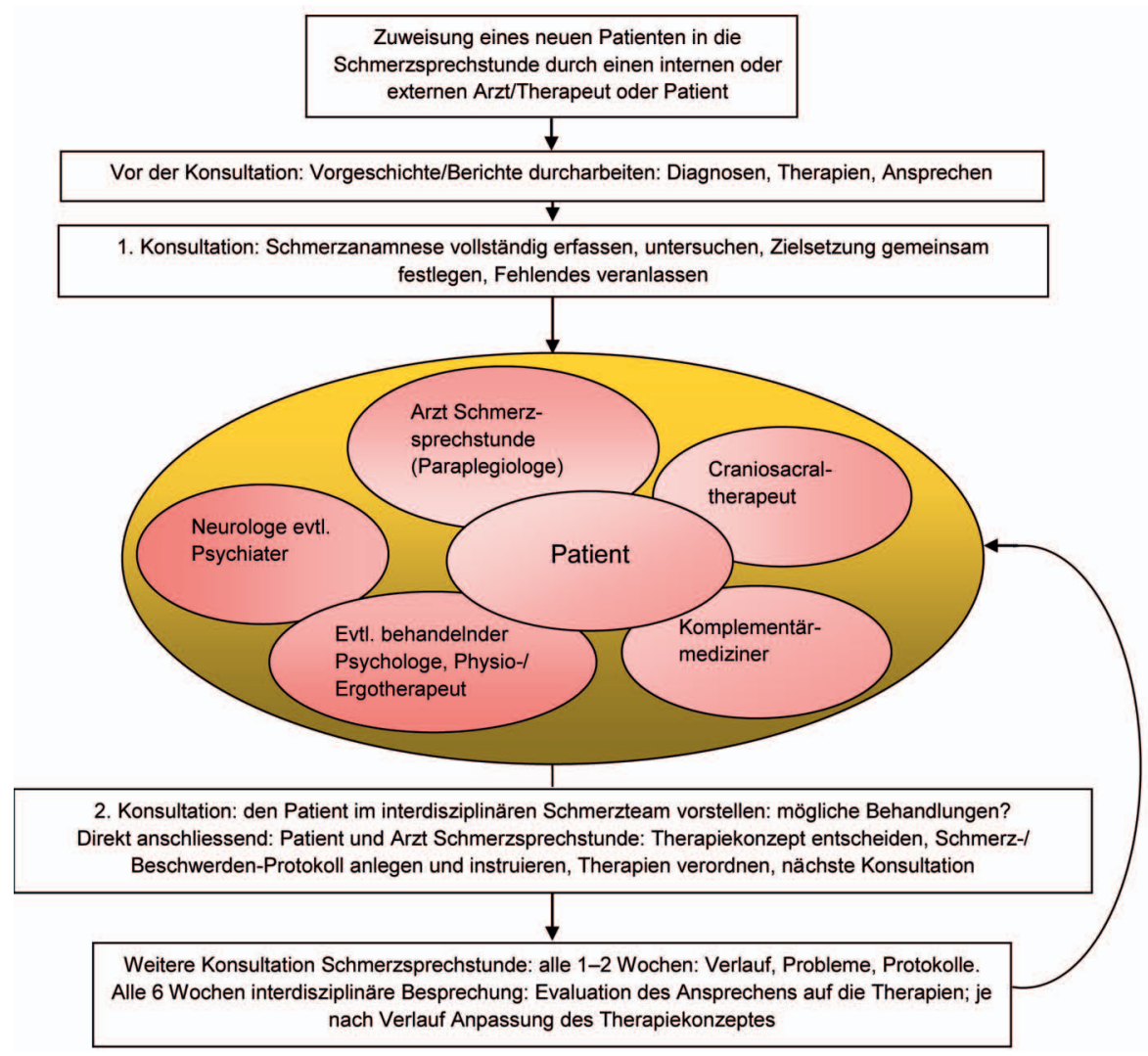

Abb. 2. Ablauf der Schmerzsprechstunde.

Abb. 3. ICF-Modell der WHO.

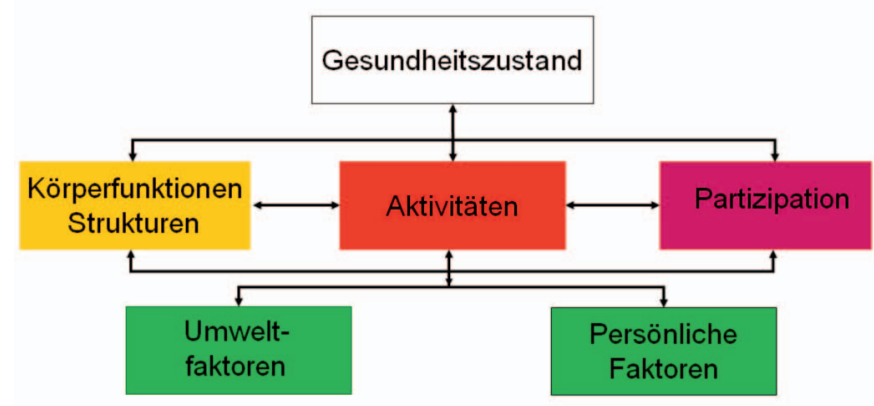

Zielklärung aufzuwenden, ermöglicht dann aber, die Patienten durch die oft langwierige Behandlung zu führen.

Grundsätzlich gilt natürlich auch für das komplementärmedizinische Angebot, dass die Behandlungschancen umso besser sind, je früher die Behandlung eingesetzt werden kann - genau wie in der klassischen Schmerzbehandlung. Begründet ist dies durch die im Gehirn einsetzenden Veränderungen bei chronischem Schmerz, wobei es neue Hinweise gibt, dass diese reversibel sein können ning, Disability and Health) der World Health Organization (WHO) auszudrücken:

Wohl liegt bei unseren Patienten mit ihren oft komplexen Schmerzsituationen meist eine Störung der Körperfunktion zugrunde, die sich beschreiben, abklären und differenziert behandeln lässt. Diese Schmerzen haben aber Auswirkungen auf die Aktivität der Patienten in ihrem Alltag, auch auf Mobilität, Selbstversorgung, die Möglichkeit, den Haushalt zu besorgen, usw. und auf die Möglichkeit, am gesellschaftlichen Leben teilzuhaben - nicht nur im Sinne der Arbeitsfähigkeit, sondern auch in Bezug auf Hobbys, kulturelles Leben, Sportverein. Und oft bestehen nebst den Schmerzen auch weitere Barrieren in der konkreten Lebensumgebung, im Kontext oder auch in der eigenen Persönlichkeit, z.B. indem es dem Betroffenen schwer fällt, Hilfe anzufordern, weil er fürchtet, zur Last zu fallen.

Aufgrund der ganzheitlichen Betrachtung des ICF (Abb. 3) zeigt sich nebst dem Schmerz auch dessen Auswirkung auf alle weiteren Körperfunktionen und Lebensbereiche [9] sowie auch der Einfluss der Umgebung auf die Betroffenen. So entsteht das vollständige Bild des Menschen mit Schmerzen und seinen Auswirkungen in der individuellen Lebenssituation [10]. Dies wiederum lässt erst die ganze Vielfalt möglicher Behandlungsansätze erkennen; allerdings gilt es dann, mit dem Betroffenen gemeinsam zu klären, welche Bereiche ihm besonders wichtig sind, und daraus festzulegen, wo es sinnvoll ist, anzusetzen, und welche Ziele erreicht werden können - und sollen.

Die verschiedenen Lösungsansätze vor sich zu sehen, ist umso wichtiger, da viele Betroffene sie durch ihr eigenes Betroffensein gar nicht erkennen können - der Schmerz überlagert alles. Darum äussern die Patienten auch meist als erstes Ziel: «der Schmerz muss weg» oder er solle «zumindest 
besser werden». Dies würde aber für die ganzheitliche Behandlung zu kurz greifen, denn inzwischen hat der Schmerz meist das Leben längst verändert, und auch wenn er weg wäre, wäre das Leben nicht mehr wie zuvor. Dies zu vermitteln und die richtigen Schritte nacheinander $\mathrm{zu}$ gehen [11, 12] sowie den langen Atem zu behalten, ist dann der Prozess in der Begleitung von Schmerzpatienten - bis die genügende Besserung erreicht ist.

\section{Literatur}

1 PJ Siddall, JD Loeser: Pain following SCI. Spinal Cord 2001;39:63-73.

2 Wasner G, Baron R: Klassifikation von Schmerzen bei Querschnittpatienten. Deutscher Schmerzkongress 2007.

3 Schleinzer W: Therapie von Schmerzen bei querschnittgelähmten Patienten mit besonderer Betonung zentraler Schmerzen. Deutscher Schmerzkongress 2007.

4 Lanz S, Maihöfner C: Symptome und pathophysiologische Mechanismen neuropathischer Schmerzsyndrome. Nervenarzt 2009;80:430-444.

5 Attal N, Backonja M, Nickolson B, Serra J: Kurswechsel beim Management neuropathischer Schmerzen. International Coalition on Neuropathic Pain, 2003.

6 Baron R, Büttner W: Diagnosen von peripheren neuropathischen Schmerzen. Deutsche Gesellschaft für Neurologie/DGN 2008.

7 Maihöfner C: CRPS und Motorik - aktuelle Erkenntnisse durch funktionelle Bildgebung. Deutscher Schmerzkongress 2007.
8 Renaud S, et al.: Statements zur Diagnostik und Therapie chronisch neuropathischer Schmerzen. Empfehlungen der Arbeitsgruppe (Special Interest Group, SIG) der Schweizerischen Gesellschaft zum Studium des Schmerzes (SGSS), 2011.

9 Stucki G, Ewert T, Cieza A: Value and application of the ICF in rehabilitation medicine. Disabil Rehabil 2002;24:932-938.

10 Biering-Sørensen F, et al.: Developing core sets for persons with spinal cord injuries based on the International Classification of Functioning, Disability and Health as a way to specify functioning. Spinal Cord 2006;44:541-546.

11 Attal N, et al.: EFNS guidelines on the pharmacological treatment of neuropathic pain: 2010 revision. Eur J Neurol 2010; 17:1113-e88.

12 Siddall PJ: Management of neuropathic pain following spinal cord injury: now and in the future. Spinal Cord 2009;47:352-359. 